\title{
Clinical and ophthalmological outcome of endoscopic transorbital surgery for cranioorbital tumors
}

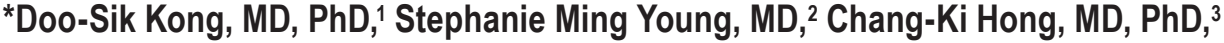 \\ Yoon-Duck Kim, MD, PhD, ${ }^{4}$ Sang Duk Hong, MD, PhD, ${ }^{5}$ Jung Won Choi, MD, PhD, ${ }^{1}$ \\ Ho Jun Seol, MD, PhD, ${ }^{1}$ Jung-II Lee, MD, PhD, ${ }^{1}$ Hyung Jin Shin, MD, PhD, ${ }^{1}$ and \\ Do-Hyun Nam, MD, PhD, ${ }^{1}$ and Kyung In Woo, MD, PhD ${ }^{4}$
}

1Department of Neurosurgery, Samsung Medical Center, Sungkyunkwan University School of Medicine, Seoul, Korea; 2Department of Ophthalmology, National University Hospital, Singapore; ${ }^{3}$ Department of Neurosurgery, Gangnam Severance Hospital, Yonsei University; ${ }^{4}$ Department of Ophthalmology, Samsung Medical Center, Sungkyunkwan University School of Medicine; and ${ }^{5}$ Department of Otorhinolaryngology-Head and Neck Surgery, Samsung Medical Center, Sungkyunkwan University School of Medicine, Seoul, Korea

OBJECTIVE Cranioorbital tumors are complex lesions that involve the deep orbit, floor of the frontal bone, and lesser and greater wing of the sphenoid bone. The purpose of this study was to describe the clinical and ophthalmological outcomes with an endoscopic transorbital approach (TOA) in the management of cranioorbital tumors involving the deep orbit and intracranial compartment.

METHODS The authors performed endoscopic TOAs via the superior eyelid crease incision in 18 patients (16 TOA alone and 2 TOA combined with a simultaneous endonasal endoscopic resection) with cranioorbital tumors from September 2016 to November 2017. There were 12 patients with sphenoorbital meningiomas. Other lesions included osteosarcoma, plasmacytoma, sebaceous gland carcinoma, intraconal schwannoma, cystic teratoma, and fibrous dysplasia. Ten patients had primary lesions and 8 patients had recurrent tumors. Thirteen patients had intradural lesions, while 5 had only extradural lesions.

RESULTS Of 18 patients, 7 patients underwent gross-total resection of the tumor and 7 patients underwent planned near-total resection of the tumor, leaving the cavernous sinus lesion. Subtotal resection was performed in 4 patients with recurrent tumors. There was no postoperative CSF leak requiring reconstruction surgery. Fourteen of 18 patients (77.8\%) had preoperative proptosis on the ipsilateral side, and all 14 patients had improvement in exophthalmos; the mean proptosis reduced from $5.7 \pm 2.7 \mathrm{~mm}$ to $1.5 \pm 1.4 \mathrm{~mm}$. However, some residual proptosis was evident in 9 of the $14(64 \%)$. Ten of 18 patients (55.6\%) had preoperative optic neuropathy, and 6 of them (60.0\%) had improvement; the median bestcorrected visual acuity improved from 20/100 to 20/40. Thirteen of 18 patients showed mild ptosis at an immediate postoperative examination, all of whom had a spontaneous and complete recovery of their ptosis during the follow-up period. Three of 7 patients showed improvement in extraocular motility after surgery.

CONCLUSIONS Endoscopic TOA can be considered as an option in the management of cranioorbital tumors involving complex anatomical areas, with acceptable sequelae and morbidity.

https://thejns.org/doi/abs/10.3171/2018.3.JNS173233

KEYWORDS transorbital surgery; cranioorbital tumor; endoscopic; oncology

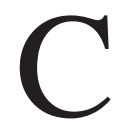
RANIOORBITAL tumors are complex lesions that involve the deep orbit, floor of the frontal bone, and lesser and greater wings of the sphenoid bone, where the skull base abuts both the anterior and middle cranial fossa. These tumors often produce significant ocu- lar manifestations, such as compressive optic neuropathy, proptosis, or limitation of extraocular movement. Multiple approaches, such as cranioorbitozygomatic surgery, have been described to access these lesions. Recently, endoscopic skull base surgery has improved the ability to ac-

ABBREVIATIONS EOM = extraocular motility; GTR = gross-total resection; NTR = near-total resection; STR = subtotal tumor resection; TOA = transorbital approach SUBMITTED December 30, 2017. ACCEPTED March 19, 2018.

INCLUDE WHEN CITING Published online September 14, 2018; DOI: 10.3171/2018.3.JNS173233.

${ }^{*}$ D.S.K., S.M.Y., and C.K.H. contributed equally to this work. 
cess complex skull base lesions. The endoscopic transorbital approach (TOA) allows access to the lateral compartment of the orbit, anterior cranial fossa, and middle cranial fossa. ${ }^{1-3,5-7,11,15,16,18,19}$ Several anatomical studies have shown that it is possible to temporarily retract the eyeballs for direct access to the sphenoid base, orbital apex, and frontal lobe and part of the skull..$^{7,8,18,21}$ Compared to the conventional transcranial approaches, endoscopic TOA has the advantages of minimal scarring limited to the eyelid crease, minimum and more direct access without the risk of cerebral retraction, and manipulation of the temporalis muscle. ${ }^{9}$ Unlike endoscopic endonasal surgery, which is limited to more medial lesions due to barriers such as the optic nerve and carotid arteries, the TOA overcomes the limitation of the surgical corridor via the endonasal route, allowing access beyond the central skull base and to more laterally extending lesions. ${ }^{6}$

To our knowledge, there have been a few papers describing the efficacy of an endoscopic TOA alone and a combined multiportal endoscopic endonasal-transorbital approach. ${ }^{3,6,19}$ In addition, there is limited literature describing a TOA to cranioorbital tumors via a superior eyelid crease incision, with specific ophthalmic outcome measures. ${ }^{1}$ The purpose of this study was to describe the clinical and ophthalmological outcomes of patients who underwent an endoscopic TOA in the management of cranioorbital tumors involving the deep orbit and intracranial compartment.

\section{Methods}

We performed a prospective surgical case series on 18 patients with cranioorbital tumors including the orbit, frontal floor, or sphenoid wings in whom an endoscopic TOA had been performed via the superior eyelid crease incision with the guidance of neuronavigation. All surgeries were performed by two neurosurgeons (D.S.K. and C.K.H.) and an oculoplastic surgeon (K.I.W.) between September 2016 and November 2017. The study was approved by the hospital's institutional review board, and it adhered to the tenets of the Declaration of Helsinki. Main outcome measures included the extent of resection, improvement of optic neuropathy, exophthalmos, and limitation of ocular motility. Optic neuropathy and limitation of ocular motility were assessed, and the degree of exophthalmos was measured with a Hertel's exophthalmometer by a specialized oculoplastic surgeon (S.M.Y.). Relative afferent pupillary defects and visual acuity were measured with the swinging light test and a visual acuity chart by two ophthalmologists (K.I.W. and S.M.Y.). Any complications related to the procedure were also noted. All postoperative MR images were obtained within 2 days of surgery. The extent of resection was interpreted by an independent radiologist. Gross-total resection (GTR) was defined as $100 \%$ tumor removal in the operation record and no remaining tumor demonstrated on postoperative MRI according to an independent radiologist. Near-total resection (NTR) was defined as greater than $90 \%$ tumor removal in the operation record or visible lesion remaining on postoperative MRI. Subtotal tumor resection (STR) was defined as less than $90 \%$ tumor removal.

\section{Surgical Technique of TOA}

Under general anesthesia, the patient was positioned supine in rigid 3-point fixation under the guidance of neuronavigation. Both eyes and face were prepared with 5\% povidone-iodine solution, and a corneal protector was placed on the ipsilateral side to avoid potential corneal damage. A skin marking of the superior eyelid crease was made at the crease line or supraciliary line for patients without an eyelid crease. After infiltration of a local anesthetic with a mixture of $2 \%$ lidocaine with 1:100,000 epinephrine and $0.5 \%$ bupivacaine, a skin incision was placed with a no. 15 Bard-Parker blade (Fig. 1 and Video 1).

VIDEO 1. Summary video clip of the operation performed in case 12. Copyright Doo-Sik Kong. Published with permission. Click here to view.

The length of the skin incision was dependent on the extent of bony removal. For instance, a half-length incision of the eyelid was sufficient to aim at opening of the lateral orbital wall alone, and a full-length eyelid incision was required for access to the frontal lobe. For removal of intraorbital tumor, we performed resection of the lateral orbital rim, the so-called extended TOA, to minimize the retraction of the globe. In such case, further extension of the incision was required. The orbicularis oculi muscle was divided along the skin incision, and gentle preseptal dissection between the septum and orbicularis muscle with Wescott scissors was carefully carried out in a superolateral direction until the lateral orbital rim was reached. Care was taken to stay on the surface of the orbital septum to avoid injury to the levator aponeurosis or preaponeurotic fat tissue. Once the periosteum was reached, incision was made and extension of the periosteum was performed along the lateral to superior orbital rim. The periorbita of the orbit was then elevated posteriorly up to the deep posterior lateral wall, taking care to prevent the herniation of periorbital fat. A $0^{\circ}$ rod-lens endoscope (Karl Storz Endoscope) was then introduced to establish visualization within the limited space along the lateral aspect of the orbit. The entire orbit was gently retracted medially, with a malleable brain retractor, to expose the lateral margin of the superior orbital fissure or tumor margin. A Silastic sheet was placed over the periorbita to prevent the iatrogenic periorbital injury. The lateral orbital wall (composed of the greater wing of the sphenoid bone) was drilled to expose the tumor without the assistance of rigid retractor. It resulted in the avoidance of excessive globe retraction. The extent of bony removal could be tailored to the surgical goal. In the case of a sphenoorbital meningioma, the lesion was first debulked using a Coblator or ultrasonic aspirator. The middle cranial fossa was exposed, and adequate hemostasis was ensured after removal of the tumor. For reconstruction of the dural defect in patients with intradural lesions, we used the double-button technique (inlay and on-lay patch) using autologous fascia or acellular allogenic dermis (Fig. 2). Additional bony reconstruction was not performed in any of the patients. Fat graft from abdominal fat tissue was performed to prevent postoperative enophthalmos in one patient. In the case of resection of the lateral orbital rim, the orbital rim was repositioned with application of a miniplate following tumor removal. Finally, the periosteum was repositioned and sutured at 

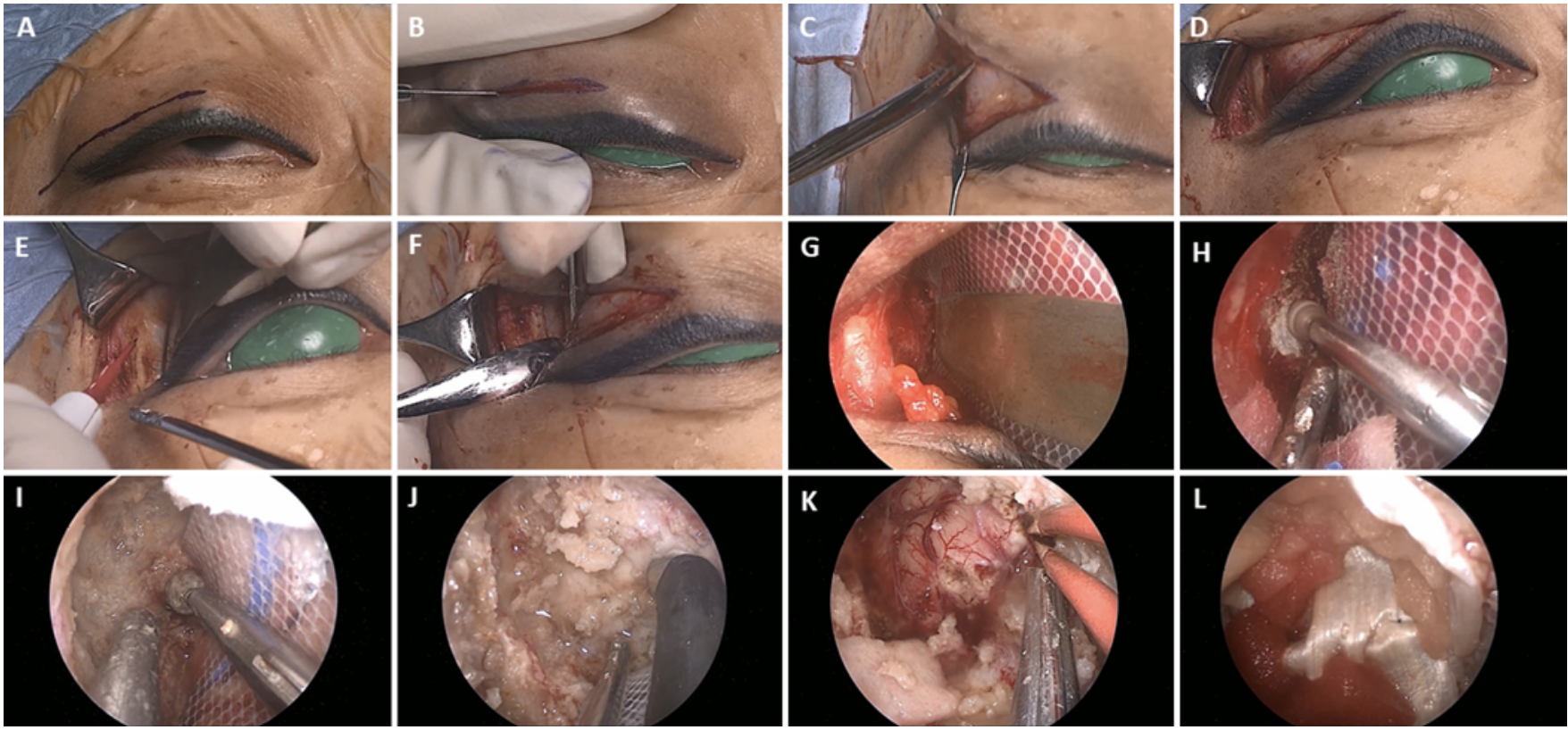

FIG. 1. Case 12: Surgical procedure. A skin marking of the superior eyelid crease (half length) is made at the crease line or supraciliary line for patients without an eyelid crease (A). After infiltration of a local anesthetic, a skin incision is made (B). Dissection between the septum and orbicularis with Wescott scissors $(\mathbf{C})$ is carried in a superolateral direction until the lateral orbital rim is reached (D). The periorbita is dissected to expose the orbital wall $(\mathbf{E})$. The periorbita is then elevated $(\mathbf{F})$ posteriorly up to the deep posterior lateral wall. An endoscope is introduced for visualization within the limited space along the lateral wall of the orbit and a Silastic sheet is used to prevent the periorbita and orbit from iatrogenic injury (G). The lateral orbital wall is drilled to gain exposure to the tumor $(\mathbf{H})$. In this case of an en plaque type of meningioma, the tumor is reduced and debulked using a high-speed diamond drill and Coblator (I and $\mathrm{J})$. The middle cranial fossa is exposed after removal of the tumor (K). The dural defect is reconstructed with the double-button technique using autologous fascia lata (L). Figure is available in color online only.

the superolateral orbital margin with 5-0 absorbable suture. The skin incision was closed with 6-0 fast-absorbing plain gut. The detailed surgical procedure is described in Videos 1 and 2.

VIDEO 2. Summary video clip of the operation performed in case 9. Copyright Doo-Sik Kong. Published with permission. Click here to view.

\section{Results}

We performed endoscopic TOA or TOA combined with the endoscopic endonasal approach in 18 patients with cranioorbital tumors. Table 1 shows the demographics and preoperative presentations of the patients. Their mean age was 51.7 years and the range was $18-73$ years. There were 4 men and 14 women. Twelve patients had sphenoorbital meningioma extending along the sphenoid ridge and orbit with or without cavernous sinus involvement; 3 patients had malignant disease (osteosarcoma, plasmacytoma, and sebaceous gland carcinoma); and 3 other patients had one of the following: intraconal schwannoma, cystic teratoma, and fibrous dysplasia. Ten patients had primary lesions and 8 patients had recurrent tumors. Thirteen tumors were intradural lesions, while 5 tumors were extradural lesions. Tumors in 8 patients involved the cavernous sinus but did not produce any neurological deficits. We performed TOA alone in 15 patients and a combined multiportal surgery (TOA and simultaneous endonasal endoscopic resection) in 3 patients with tumor extending into the infratemporal fossa or medial side to the orbit. The mean follow-up duration was $5.3 \pm 3.7$ months (range $1-11$ months).

\section{Clinical Outcome}

Of 18 patients, 7 patients underwent GTR of the tumor and 7 patients had NTR of the tumor, leaving a portion of the lesion in the cavernous sinus (Figs. 3 and 4). STR was performed in 4 patients with recurrent tumors. All patients who underwent NTR or STR received subsequent Gamma Knife radiosurgery for the remaining lesions. Overall, 14 of 18 patients $(77.8 \%)$ had GTR or NTR of the tumor. We performed lumbar drainage of CSF for 3 days postoperatively in the first 3 patients who underwent TOA, but did not perform CSF diversion in 15 other patients. There were no
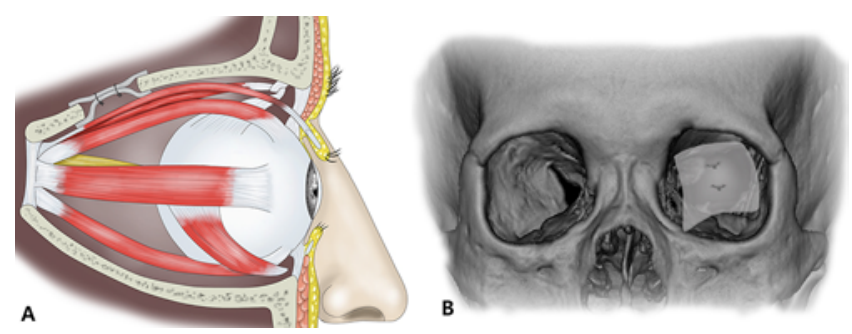

FIG. 2. Illustration showing the dural reconstruction method (A and B). Copyright Samsung Medical Center. Published with permission. Figure is available in color online only. 
Kong et al.

TABLE 1. Patient demographics and preoperative ophthalmological findings

\begin{tabular}{|c|c|c|c|c|c|c|c|c|c|}
\hline Case No. & Age (yrs) & Sex & Diagnosis & Dural Involvement & Primary/Recurrent & CS & Optic Neuropathy & Proptosis & EOM Limitation \\
\hline 1 & 64 & M & Meningothelial meningioma & Intradural & Primary & No & Yes & Yes & No \\
\hline 2 & 43 & M & Sebaceous gland carcinoma & Extradural & Primary & No & No & Yes & Yes \\
\hline 3 & 40 & $M$ & Cystic teratoma & Extradural & Recurrent & No & No & Yes & Yes \\
\hline 4 & 51 & $\mathrm{~F}$ & Meningothelial meningioma & Intradural & Recurrent & Yes & Yes & Yes & Yes \\
\hline 5 & 56 & $\mathrm{~F}$ & Atypical meningioma & Intradural & Primary & Yes & No & Yes & No \\
\hline 6 & 40 & $\mathrm{~F}$ & Intraconal schwannoma & Extradural & Primary & No & Yes & No & No \\
\hline 7 & 23 & $\mathrm{~F}$ & Osteosarcoma & Intradural & Primary & No & Yes & Yes & Yes \\
\hline 8 & 55 & $\mathrm{~F}$ & Plasmacytoma & Extradural & Primary & No & No & Yes & No \\
\hline 9 & 52 & $\mathrm{~F}$ & Meningothelial meningioma & Intradural & Recurrent & Yes & Yes & Yes & No \\
\hline 10 & 52 & $\mathrm{~F}$ & Transitional meningioma & Intradural & Recurrent & Yes & Yes & Yes & No \\
\hline 11 & 73 & $\mathrm{~F}$ & Atypical meningioma & Intradural & Primary & Yes & No & No & No \\
\hline 12 & 38 & $\mathrm{~F}$ & Transitional meningioma & Intradural & Recurrent & No & No & Yes & No \\
\hline 13 & 45 & $\mathrm{~F}$ & Transitional meningioma & Intradural & Recurrent & Yes & Yes & Yes & Yes \\
\hline 14 & 59 & $\mathrm{~F}$ & Meningothelial meningioma & Intradural & Primary & Yes & No & Yes & No \\
\hline 15 & 18 & M & Fibrous dysplasia & Extradural & Primary & No & Yes & Yes & Yes \\
\hline 16 & 58 & $\mathrm{~F}$ & Meningothelial meningioma & Intradural & Primary & Yes & Yes & Yes & Yes \\
\hline 17 & 65 & $\mathrm{~F}$ & Meningothelial meningioma & Intradural & Primary & No & No & No & No \\
\hline 18 & 60 & $\mathrm{~F}$ & Meningothelial meningioma & Intradural & Primary & No & Yes & No & No \\
\hline
\end{tabular}

CS = cavernous sinus.
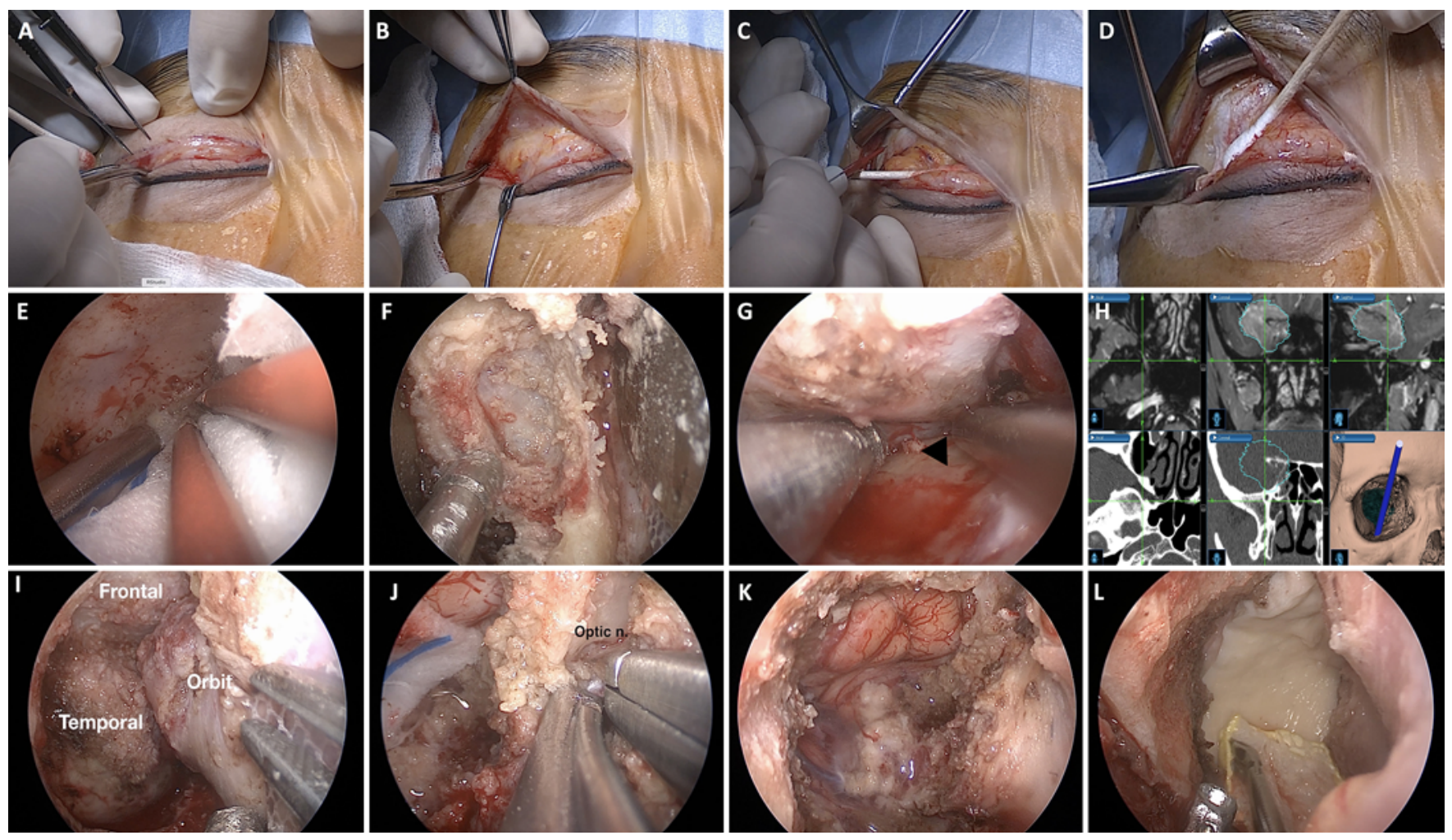

FIG. 3. Case 9: Surgical procedure. A skin incision on the superior eyelid crease (full length) is made to approach the orbital roof (A). The preseptal approach is carefully carried out in a superolateral direction until the lateral orbital rim is reached (B). The periosteum is incised with a monopolar coagulator along the orbital rim (C). The periosteum is elevated from the lateral orbital rim (D). After removal of the lateral orbital rim (not shown), the periorbita is dissected to expose the tumor margin (E). The lateral orbital wall is drilled to expose the middle fossa dura $(\mathbf{F})$. The foramen rotundum (indicator) can be identified under neuronavigation $(\mathbf{G}$ and $\mathbf{H})$. After adequate removal of the sphenoid bone and frontal floor, the tumor margin is fully exposed creating one surgical view (I). After debulking the tumor, the optic nerve can be seen $(\mathbf{J})$. A tumor portion involving the cavernous sinus is left in place $(\mathbf{K})$. A wide dural defect is reconstructed using the double-button technique with allogenic dermis (L). Figure is available in color online only. 

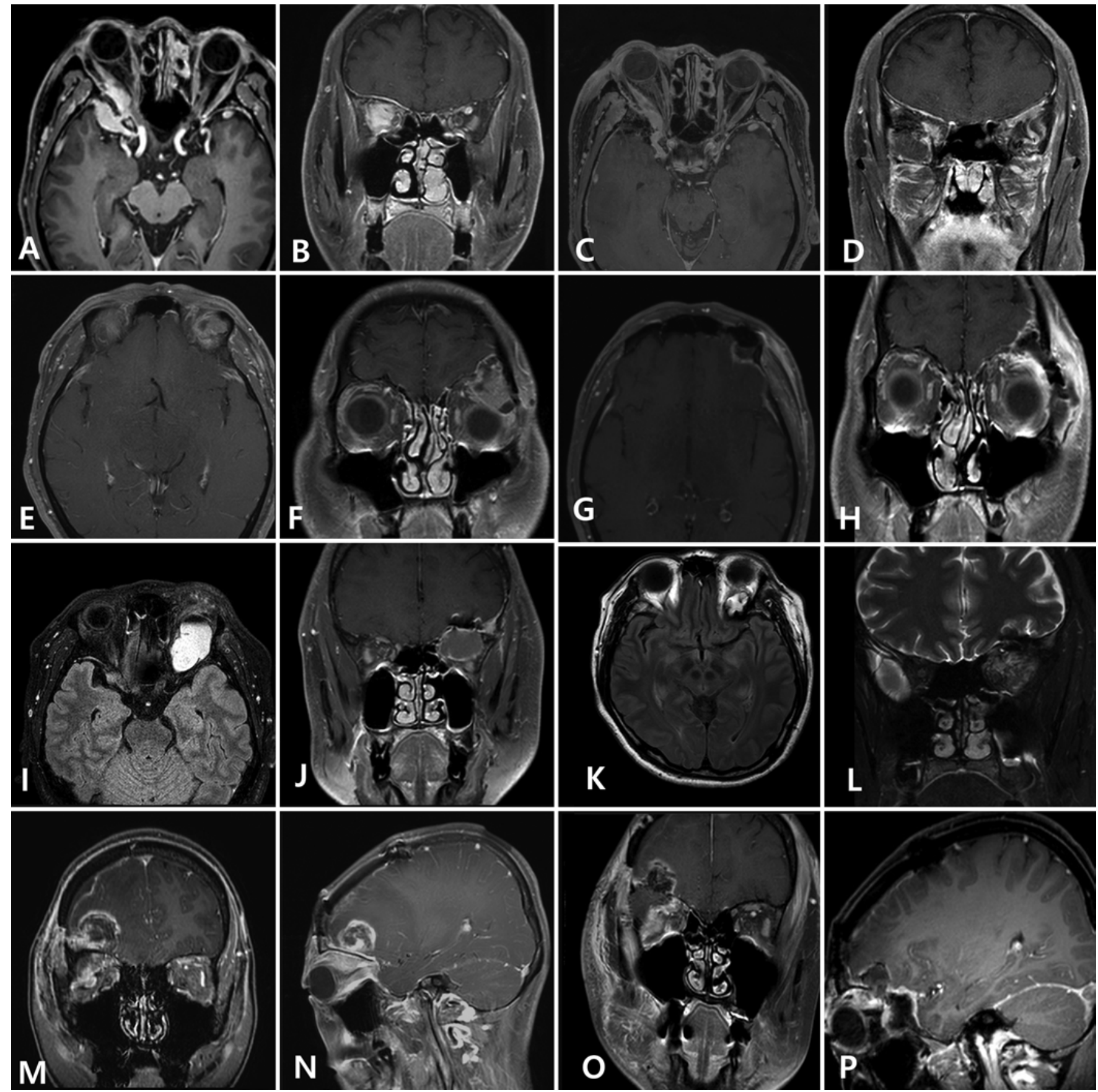

FIG. 4. Cases 1-3 and 7. Case 1: Sphenoorbital meningioma shown on preoperative MR images (A and B) and, after NTR, on postoperative MR images ( $C$ and $\mathbf{D})$. Case 2: Sebaceous gland carcinoma involving the frontoorbital bone demonstrated on preoperative MR images (E and F) and, after GTR, on postoperative MR images ( $\mathbf{G}$ and $\mathbf{H})$. Case 3: Recurrent cystic teratoma located in the orbit shown on preoperative MR images (I and $\mathbf{J}$ ) and, after NTR, on postoperative MR images (K and L). Case 7: Recurrent metastatic osteosarcoma involving the frontal lobe and orbit demonstrated on preoperative MR images ( $M$ and $\mathbf{N}$ ) and, after GTR, on postoperative $\mathrm{MR}$ images $(\mathbf{O}$ and $\mathbf{P})$.

postoperative CSF leaks requiring reconstruction surgery. Postoperatively, 2 of 18 patients demonstrated early postoperative periorbital swelling due to CSF collection. However, these patients had improvement of their swelling within 2 weeks by slight compression of the globe with an eye patch or shield. Thirteen of 18 patients showed mild ptosis in the early postoperative period in all of whom spontaneous and complete recovery of ptosis occurred during the follow-up period. No patient complained of postoperative headache. To treat the remaining tumor in the patients with meningioma, Gamma Knife radiosurgery was performed postoperatively to prevent regrowth of the tumor. 


\section{Ophthalmological Outcome}

Fourteen of 18 patients (77.8\%) had preoperative proptosis on the ipsilateral side, and 10 patients $(55.6 \%)$ had preoperative compressive optic neuropathy. The mean displacement of preoperative proptosis was $5.7 \pm 2.7 \mathrm{~mm}$ (range $2-10 \mathrm{~mm}$ ) (Table 2). All 14 patients had improvement in exophthalmos (Fig. 5). The mean proptosis postsurgery decreased to $1.5 \pm 1.4 \mathrm{~mm}$ (range $0-4 \mathrm{~mm}$ ). Of the 10 patients with preoperative optic neuropathy, 6 patients $(60.0 \%)$ had improvement, with resolution of their relative afferent pupillary defect (Fig. 6). The median value of best-corrected visual acuity was improved, from 20/100 (range finger counting $10 \mathrm{~cm}-20 / 32$ ) to 20/40 (range finger counting $10 \mathrm{~cm}-20 / 25)$ after surgery. Seven patients (38.9\%) showed extraocular motility (EOM) limitation preoperatively; one patient had strabismus at the primary position with peripheral EOM limitation; and the remaining patients showed peripheral limitation in horizontal and/ or vertical gaze. Three of 7 patients showed improvement in EOM after surgery. In contrast, 2 other patients without preoperative abnormality of motility presented with new-onset lateral rectus paresis immediately after surgery, which resolved during the follow-up period. Thirteen of 18 patients had mild ptosis immediately after surgery, but this improved completely in all patients within 4 weeks. During follow-up ophthalmic examination, no patient was shown to have visual deterioration or to have worsened proptosis.

\section{Discussion}

Cranioorbital tumors are anatomically complex lesions involving multiple compartments, such as the orbit and cranial vault. These tumors sometimes show a locally invasive en plaque pattern, spreading to surrounding structures, including the superior orbital fissure, cavernous sinus, periorbita, or adjacent dura mater. The tumor can also result in hyperostosis of the greater wing of the sphenoid bone, resulting in orbital compression. They commonly include sphenoorbital meningiomas and complicated orbital tumors with intracranial extension, such as schwannoma, cystic teratoma, and other malignancies. ${ }^{10,13}$ The main clinical manifestations include progressive proptosis, decreased visual acuity, partial ptosis, and ocular motility restriction.

Traditionally, a conventional cranioorbital craniotomy and removal of the zygomatic process would provide the best approach for tumor removal or debulking in this area. ${ }^{10,12,13}$ However, transcranial approaches to the orbit often require complex procedures that have risks related to the craniotomy, cerebral retraction, and violation of the cranial space. ${ }^{11}$ In addition, they also lead to excessive retraction of the temporalis muscle, potentially resulting in the risk of temporalis muscle atrophy. Recently, it had been emphasized that postoperative morbidity is minimized and cosmetic problems are reduced by using endoscopic approaches. Endoscopic approaches have significantly reduced the morbidity of open surgical procedures and have become as effective as traditional open surgery in selected cases. ${ }^{17}$ Various endoscopic approaches to the orbit have been described in the literature and are typically performed via an endonasal route. ${ }^{14,17,20}$ However, the main limitations of endoscopic endonasal approaches include limited working space when multiple instruments are used and a surgical corridor that is focused on the area between the orbits. ${ }^{16}$ Pathology located within or extending into the lateral part of the orbit and retroorbital space can create additional challenges in terms of surgical access.

The endoscopic TOA is a novel technique that has overcome the limited endonasal surgical corridor, and it provides access to the contents of the orbit as well as the intracranial compartment, including the anterior and middle fossa and lateral cavernous sinus., ${ }^{5,6,16,19}$ To our knowledge, while there have been a few papers on the TOA for orbital lesions, $, 3,6,19$ the literature describing a purely TOA for cranioorbital tumors via a superior eyelid crease incision, with specific ophthalmic outcome measurement, is limited. ${ }^{1}$ Our study demonstrates that, via a superior lid crease incision (either the lateral half or full length of the crease), we can successfully remove cranioorbital tumors with minimal postoperative morbidity and excellent improvement in ophthalmological outcome. In particular, the locally invasive nature of en plaque sphenoid wing meningiomas requires a wide exposure to remove all involved structures, which usually include the greater and lesser wings of the sphenoid bone, orbit, and infratemporal fossa. ${ }^{4,13}$ The transorbital route overcomes the limitations of the open transcranial approach for tumors involving multiple compartments and allows more direct access to the lesions and better visualization of tumors invading the lateral part of the sphenoid body at the origin of the greater wing and cavernous sinus. ${ }^{4}$ In this study, only 4 of 12 patients (33.3\%) with sphenoorbital meningioma underwent GTR. It was attributable to the en plaque growth pattern, the basic property of sphenoorbital meningioma. In addition, 8 of 12 patients (66.7\%) showed preoperative cavernous sinus involvement. Without preoperative neurological deficits such as cranial nerve injury in cases in which the tumor involved the cavernous sinus, the cavernous sinus portion of the tumor was left in place to avoid the risk of causing postoperative neurological deficits; thus, a conservative surgical strategy concerning the cavernous portion was chosen in this study. Our series has shown that in most cases, the extent of resection was comparable to that of a conventional approach, and preoperative ophthalmic manifestations such as proptosis or compressive optic neuropathy improved after surgery.

In this study, compressive optic neuropathy recovered in $60 \%$ of patients. The apical compression caused by cranioorbital tumors could be relieved with tumor removal and simultaneous decompression of thickened lateral orbital wall. However, the patients who had profound optic atrophy, from a history of long-lasting tumor or previous surgeries, did not show visual improvement even after the TOA. Two patients developed lateral rectus paresis after TOA, which might be related to inflammation, swelling of the lateral rectus, and/or transient abducent nerve paresis. A further evaluation with a longer follow-up should be required. Otherwise, preexisting EOM limitations recovered to varying degrees in the other patients, and a postoperative mild ptosis in 13 patients also resolved within the follow-up period. Therefore, no significant adverse effects were found in patients in whom the TOA was used 


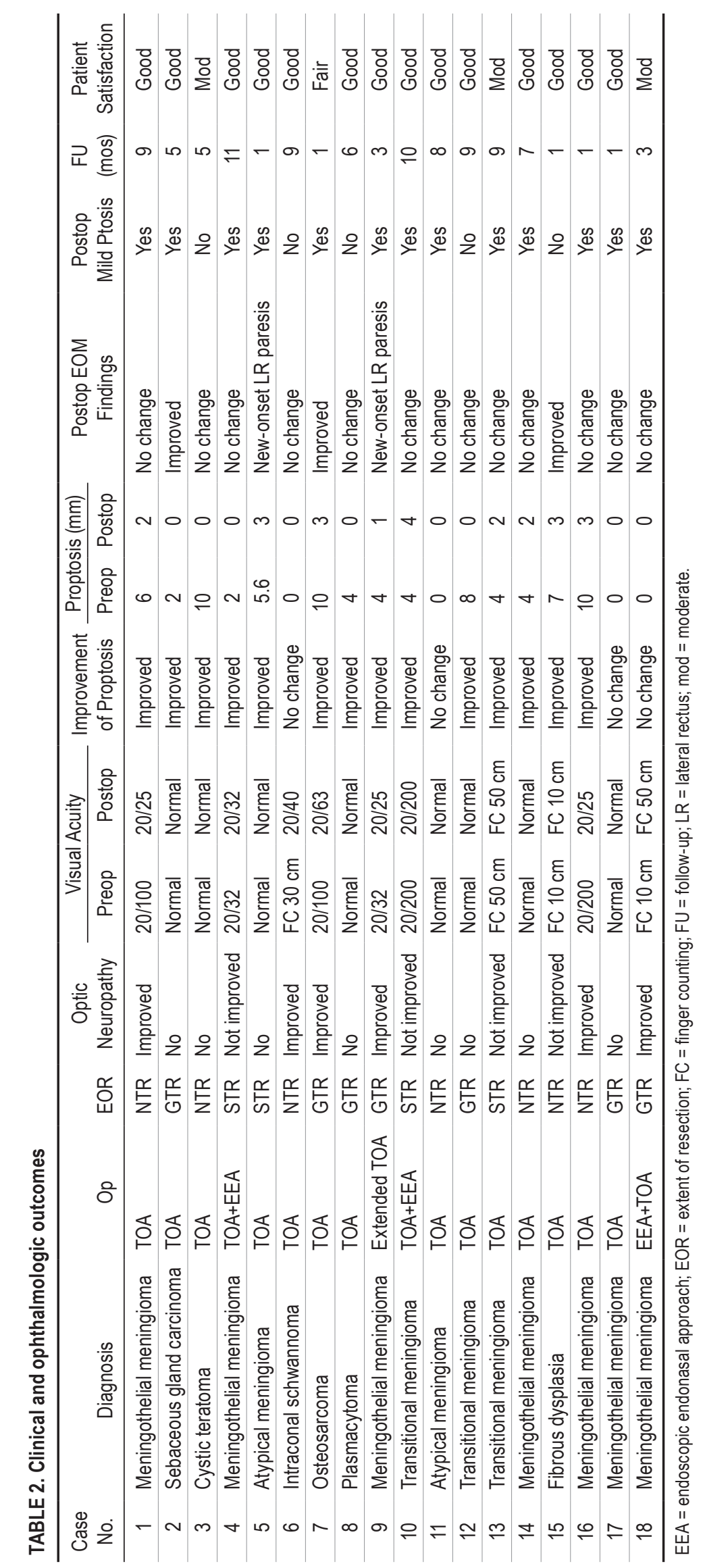




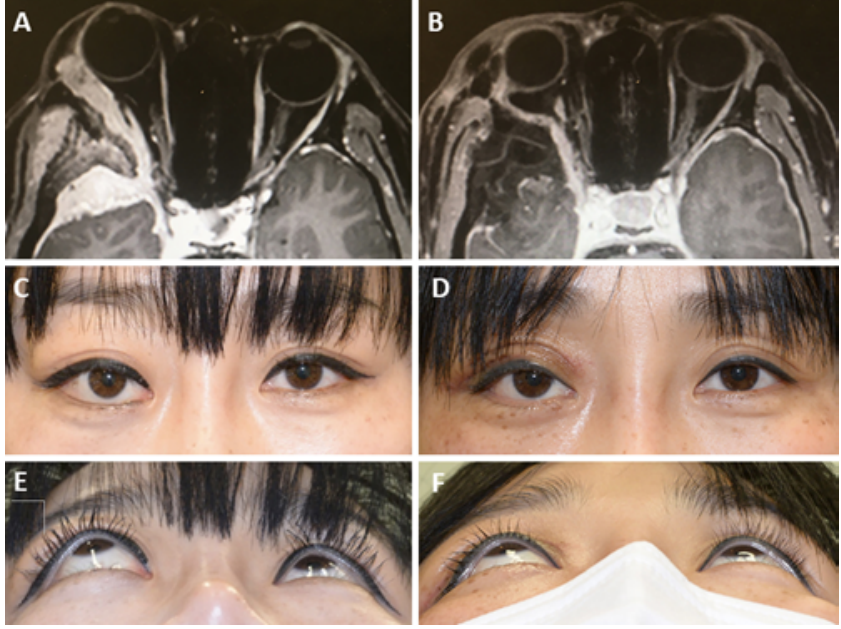

FIG. 5. Case 12: Illustrative case. A 38-year-old woman, with a history of receiving Gamma Knife radiosurgery for a sphenoid wing meningioma involving the lateral orbit, presented with progressive proptosis of $8 \mathrm{~mm}$ (A, C, and E). Preoperative MRI showed thickened hyperostosis of the sphenoid bone caused by tumor invasion. She underwent endoscopic transorbital surgery via a superior eyelid crease approach. Postoperative MR image demonstrates GTR of the lesion (B). The patient was discharged 3 days after surgery with very limited edema around the upper eyelid; her proptosis had completely resolved and she had no ophthalmic-related complications ( $\mathbf{D}$ and $\mathbf{F}$ ). Figure is available in color online only. for cranioorbital tumors from an ophthalmological point of view.

To date, the TOA has been shown to achieve successful resection of tumors without significant morbidities such as postoperative CSF leaks or ophthalmic complications. The TOA requires a thorough knowledge of the orbit and skull base anatomy. The advantages of the TOA over traditional transcranial approaches include an absence of a cranial incision, limited or absent brain retraction, no manipulation of the temporalis muscle, no removal of temporal bone base, less risk of postoperative CSF leaks, and acceptable sequelae and reduced morbidity. ${ }^{5,6,16}$ In the literature, there has been no report of postoperative CSF leak after a TOA because of the barrier provided by the globe; however, in most series, the lesions were limited to the extradural lesions or CSF leaks after trauma..$^{9,11,16,19}$ In our series, we had 2 cases of CSF leaks immediately after removal of intradural tumors. We found that simple compression of the globe with an eyelid shield or patch improved periorbital swelling caused by the postoperative CSF leaks.

Limitations of our study include the relatively small number of patients and the short follow-up period. Since this novel approach was applied in Korea only from September 2016, the number of suitable patients with available ophthalmic follow-up was limited. As with any new and exciting field, one does not expect immediate acceptance and broad adoption of this technique. Nevertheless, this
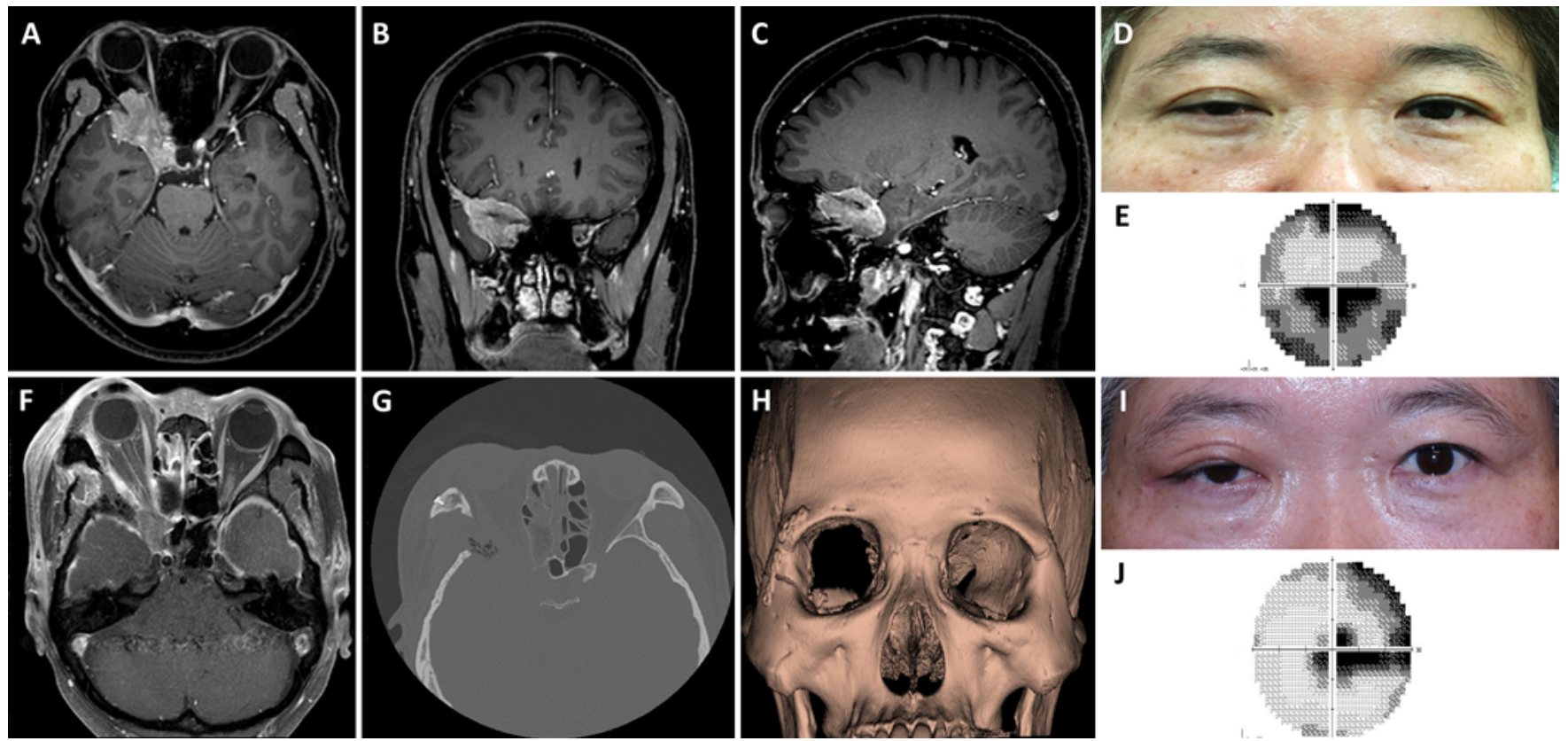

FIG. 6. Case 9: Illustrative case. A 52-year-old woman showed progressive proptosis of $4 \mathrm{~mm}$, a relative afferent pupillary defect, and compressive optic neuropathy (best-corrected visual acuity [BCVA] 20/32) on the ipsilateral side (D and E). Preoperative MR images showed that the tumor involved the orbital globe, periorbita, the sphenoidal ridge, and the temporal tip (A-C). The tumor widened the superior orbital fissure (SOF), extending the cavernous sinus through the SOF. She had no other cranial nerve deficits. She underwent an extended endoscopic transorbital resection of the tumor. To avoid excessive retraction of the globe, the lateral rim of the orbit was removed to allow removal of the large intraorbital portion of the tumor. NTR of the tumor was performed, leaving the cavernous sinus portion of the lesion (F). Postoperative CT scans showed a large skull defect of the superior and lateral wall of orbit (G and $\mathbf{H})$. Immediately after surgery, she had mild ptosis and periorbital swelling (I); however, the ptosis recovered within 4 weeks. Postoperatively, her decreased visual acuity and field defect were improved (BCVA 20/25 [J]). Figure is available in color online only. 
approach should be considered as an option that can be used when faced with appropriate situations and can be an alternative to the traditional transcranial approach for cranioorbital tumors extending into the surrounding structures.

\section{Conclusions}

Endoscopic TOA is an option in the management of cranioorbital tumors involving complex anatomical structures, with satisfactory results and morbidity in relation to the traditional approaches. Further studies are required to validate this surgical technique and assess its equivalency with conventional transcranial approaches.

\section{Acknowledgments}

This study is supported by the National Research Foundation of Korea by the Ministry of Science, ICT and Future Planning (MSIP) of Korea and grants (NRF-2015M3A9A7029740, NRF-2015M3C9A1044522, and NRF-2015M3A9B5053642) of the National Research Foundation funded by MSIP of Korea, and by a Samsung Medical Center grant.

\section{References}

1. Almeida JP, Omay SB, Shetty SR, Chen YN, Ruiz-Treviño AS, Liang B, et al: Transorbital endoscopic eyelid approach for resection of sphenoorbital meningiomas with predominant hyperostosis: report of 2 cases. J Neurosurg 128:18851895,2018

2. Almeida JP, Ruiz-Treviño AS, Shetty SR, Omay SB, Anand VK, Schwartz TH: Transorbital endoscopic approach for exposure of the sylvian fissure, middle cerebral artery and crural cistern: an anatomical study. Acta Neurochir (Wien) 159:1893-1907, 2017

3. Bly RA, Ramakrishna R, Ferreira M, Moe KS: Lateral transorbital neuroendoscopic approach to the lateral cavernous sinus. J Neurol Surg B Skull Base 75:11-17, 2014

4. Bonnal J, Thibaut A, Brotchi J, Born J: Invading meningiomas of the sphenoid ridge. J Neurosurg 53:587-599, 1980

5. Dallan I, Caniglia M, Turri-Zanoni M, Prevedello DM, DE Notaris M, Battaglia P, et al: Transorbital superior eyelid endoscopic approach to the temporal lobe. J Neurosurg Sci 62:369-372, 2018

6. Dallan I, Castelnuovo P, Locatelli D, Turri-Zanoni M, AlQahtani A, Battaglia P, et al: Multiportal combined transorbital transnasal endoscopic approach for the management of selected skull base lesions: preliminary experience. World Neurosurg 84:97-107, 2015

7. Di Somma A, Andaluz N, Cavallo LM, de Notaris M, Dallan I, Solari D, et al: Endoscopic transorbital superior eyelid approach: anatomical study from a neurosurgical perspective. J Neurosurg [epub ahead of print December 15, 2017. DOI: 10.3171/2017.4.JNS162749

8. Ferrari M, Schreiber A, Mattavelli D, Belotti F, Rampinelli V, Lancini D, et al: The inferolateral transorbital endoscopic approach: a preclinical anatomic study. World Neurosurg 90:403-413, 2016

9. Goldberg RA, Shorr N, Arnold AC, Garcia GH: Deep transorbital approach to the apex and cavernous sinus. Ophthal Plast Reconstr Surg 14:336-341, 1998

10. Jian T, Sun F, Tang D, Wang S, Wu T, Zhao L: Clinical analysis of transcranial orbitotomy approach on cranio-orbital tumors. J Craniofac Surg 26:441-446, 2015

11. Lew H, Rootman DB, Nassiri N, Goh A, Goldberg RA: Transorbital approach without craniotomy to orbital tumors with extradural intracranial extension. Orbit 33:343-351, 2014

12. Maroon JC, Kennerdell JS: Surgical approaches to the orbit. Indications and techniques. J Neurosurg 60:1226-1235, 1984

13. McDermott MW, Durity FA, Rootman J, Woodhurst WB: Combined frontotemporal-orbitozygomatic approach for tumors of the sphenoid wing and orbit. Neurosurgery 26:107116, 1990

14. McKinney KA, Snyderman CH, Carrau RL, Germanwala AV, Prevedello DM, Stefko ST, et al: Seeing the light: endoscopic endonasal intraconal orbital tumor surgery. Otolaryngol Head Neck Surg 143:699-701, 2010

15. Mishra A: Transorbital approach to infratemporal fossa: novel technique. J Laryngol Otol 125:638-642, 2011

16. Moe KS, Bergeron CM, Ellenbogen RG: Transorbital neuroendoscopic surgery. Neurosurgery 67 (3 Suppl Operative):ons16-ons28, 2010

17. Nicolai $P$, Battaglia $P$, Bignami $M$, Bolzoni Villaret $A$, Delù $\mathrm{G}$, Khrais T, et al: Endoscopic surgery for malignant tumors of the sinonasal tract and adjacent skull base: a 10-year experience. Am J Rhinol 22:308-316, 2008

18. Priddy BH, Nunes CF, Beer-Furlan A, Carrau R, Dallan I, Prevedello DM: A side door to Meckel's cave: anatomic feasibility study for the lateral transorbital approach. Oper Neurosurg (Hagerstown) 13:614-621, 2017

19. Ramakrishna R, Kim LJ, Bly RA, Moe K, Ferreira M Jr: Transorbital neuroendoscopic surgery for the treatment of skull base lesions. J Clin Neurosci 24:99-104, 2016

20. Sethi DS, Lau DP: Endoscopic management of orbital apex lesions. Am J Rhinol 11:449-455, 1997

21. Sonig A, Nanda A: Transorbital approach to the anterior cranial skull base. World Neurosurg 80:810-812, 2013

\section{Disclosures}

The authors report no conflict of interest concerning the materials or methods used in this study or the findings specified in this paper.

\section{Author Contributions}

Conception and design: Woo, Kong. Acquisition of data: Kong, CK Hong. Analysis and interpretation of data: Kong, Seol. Drafting the article: Woo, Kong, Young, Choi. Critically revising the article: Kim, SD Hong, Choi. Reviewed submitted version of manuscript: Woo, Kong. Approved the final version of the manuscript on behalf of all authors: Woo. Statistical analysis: Lee. Administrative/technical/material support: Kim, Shin, Nam. Study supervision: SD Hong.

\section{Supplemental Information \\ Videos}

Video 1. https://vimeo.com/268999863.

Video 2. https://vimeo.com/268999897.

\section{Previous Presentations}

The material in this paper was presented in poster form at the European Society of Ophthalmic Plastic and Reconstructive Surgery Meeting, Stockholm, Sweden, 2017.

\section{Correspondence}

Kyung In Woo: Samsung Medical Center, Sungkyunkwan University School of Medicine, Seoul, Korea. eyeminded@skku.edu. 